crystals of the hormone at least one of these metals must be present in the animal material (beef pancreas) from which the insulin is extracted. In a paper read at this year's meeting of the Society, Drs. Scott and Fisher reported on the preparation of nickel-insulin crystals; these were found to contain about 0.4 per cent of nickel. Dr. Scott's researches have an important practical bearing for it was soon found that zinc, like protamine (a protein material obtained from salmon sperm), prolongs the action of insulin. The improved product, protamine-zine insulin, is now in general use for the treatment of diabetes. This preparation has the great advantage over the older ones in that, owing to its prolonged action, less frequent dosage is required to control the disease-a fact cordially welcomed by the diabetic patient.

An interesting series of experiments on coronary artery thrombosis was described in a paper by Dr. C. H. Best and Dr. D. Y. Solandt. Coronary thrombosis was produced in animals by the injection of a chemical (sodium ricinoleate) into the neighbourhood of one of the main arteries. The electrical changes in the heart during the period of survival and the microscopical appearance of the heart muscle after death were studied.

The researches of Dr. P. J. Moloney and his colleagues in the Connaught Laboratories, as reported in a paper entitled "The Detoxifying Action of Human Bile", reveal the fact that diphtheria toxin is innocuous after being treated with human bile.

Drs. W. R. Campbell and M. I. Hanna have found that the proportions of the different proteins in the plasma of all healthy persons are approximately the same, nor are they altered in most diseases. In some instances, however, they vary in such a way from that seen in health as to be of valuable assistance in recognizing certain diseases.

Dr. J. K. W. Willard, in the course of some experiments in British Columbia, accidentally injected some plant hormone into his hand. A tumour developed near the tiny wound. Fortunately it was an innocent growth.

\title{
Astronomy's Debt to Photography
}

$T^{\prime}$ HE Photographic Journal of April contains the thirty-third Traill Taylor Memorial Lecture entitled "Photography in the Exploration of the Universe" which was delivered by the Astronomer Royal, Dr. H. Spencer Jones, on October 19 last when he was presented with the Traill Taylor Memorial Medal of the Royal Photographic Society.

Dr. Spencer Jones began by describing the modern photographic method of determining the distances of the nearer stars. From a single plate taken with the Greenwich 26-inch refractor, giving a scale of $30^{\prime \prime}=1 \mathrm{~mm}$., the probable error of the position of a star whose parallax is being determined is about $0.024^{\prime \prime}$ which is equivalent to about $0.0008 \mathrm{~mm}$. The probable error produced by local distortions in the gelatin film does not usually exceed $0.0003 \mathrm{~mm}$., a fact which shows how eminently suitable the photographic plate is for the measurement of minute parallactic displacements given by the changing viewpoint of the earth in its annual journey around the sun. The only visual method of parallax determination comparable in accuracy with photographic methods is that of the heliometer, which in the hands of Bessel one hundred years ago yielded with considerable accuracy one of the first three stellar parallaxes to be successfully determined. It is estimated, however, that the output of results by photographic methods is increased by a factor of 15 , since the actual micrometric measures are made at any convenient time subsequent to the taking of the photograph.

At stellar distances equivalent to 400 light years, the trigonometrical method of measuring the distances of the stars breaks down, because the quantities to be measured become increasingly smaller than the probable errors of measurement, microscopic though the probable errors have been reduced to by the most scrupulous attention to every detail. For the determination of stellar distances beyond what is merely the fringe of near-by space, the astronomer makes use of the unique properties of the Cepheid variable stars, which are found not only in the star clusters of our own stellar system (some 120,000 light years in diameter) but are recognizable in the nearer and resolvable extra-galactic nebulæ whose unit of distance from us is a million light years. The discovery of the period-luminosity relation (which makes the Cepheids criteria of distance), announced by Miss Leavitt in 1912 and extended by Hertzsprung and Shapley, was made possible by a series of photographs of the Magellanic Clouds. The yet greater distances of the spiral nebulæ whose stellar structure is beyond resolution into individual stars can be determined by extrapolating the empirical relation found between distance and radial velocity, given by Doppler shifts, of the nearer systems. The photography of the spectra of these faint objects ranging up to 250 million light years away is a veritable tour de force. At the focus of the telescope their images are invisible, and the setting of the slit of the spectrograph has to be made in each case by means of a near-by star in the field of view whose angular distance from the nucleus of the nebula is obtainable from long-exposure direct photographs.

Honours in this triumph of observation have, of course, to be shared between the photographic plate, the giant telescopes employed, the special camera lens (such as the Rayton or the Bracey lens) and the ingenuity and patience of the astronomer. From statistical results of apparent magnitudes, it is deduced that the most distant systems that can be recorded on long-exposure direct photographs, on special emulsions of high-threshold sensitivity, taken with the 100 -in. reflector at Mount Wilson, are at a distance of about 500 million light years. Within a sphere whose radius is equal to this limiting distance, it is estimated by Hubble from counts taken from photographs that there are about 100 million stellar systems--veritable universes that are units of the cosmos. A number of slides shown during the lecture and in part reproduced in the article, illustrate the perfection of results given by the photographic plate in recording the Milky Way, gaseous nebulæ, star clouds and individual star clusters, and the remote extra-galactic nebulæ. 